\title{
Demokratie im Zeitalter der Globalisierung. Ein Essay
}

\author{
Anton Pelinka1,* \\ 1 Nationalism Studies Program and Department of Political Science, Central European University, Budapest \\ * E-Mail: PelinkaA@ceu.edu
}

\section{Zusammenfassung}

Der Diskussionsbeitrag beschreibt den Megatrend Demokratisierung in Verbindung mit einer permanenten Ausweitung des „demos“ in Form der Inklusion von gesellschaftlichen Teilen, die davor aus Gründen des Geschlechts, der Ethnizität („Rasse“) und/oder der religiösen oder auch politischen Überzeugung exkludiert waren. Demokratien - definiert nach den konzeptionellen Kriterien Schumpeters und Dahls - werden aber nunmehr durch die Implikationen des wachsenden Ungleichgewichts zwischen einer globalisierten Ökonomie und der abnehmenden Fähigkeiten einer staatlich limitierten Demokratien herausgefordert. Die Folge ist eine abnehmende Politikfähigkeit der Nationalstaaten und damit einer ausschließlich staatlich definierten Demokratie.

\section{Schlüsselwörter}

Demokratietheorie, Demos: Inklusion und Exklusion, Globalisierung, Gleichgewicht zwischen Freiheit und Gleichheit, nationale und/oder globale Gerechtigkeit

\section{Democracy in Times of Globalization}

\section{Abstract}

The discussion article argues that the process of democratization has been based on an ongoing enlargement of the "demos" by including segments of the society which had been excluded in the past, justified by gender, ethnicity ("race") and/or religious or political beliefs. In the established democracies - democracies according to the concepts of Schumpeter and Dahl - this process has come almost to an end. But as globalization reduces more and more the political ability of the traditional nation state to define and implement efficient policies, democracy faces a new challenge: How to deal with the growing imbalance between a globalized economy and democratic political systems, restricted in their ability to act by the limitations of nation states?

\section{Keywords}

Democratic Theory, Demos: Inclusion and Exclusion, Globalization, Balance between Liberty and Equality, national and/or global Justice

The author has declared that no competing interests exist. 


\section{Die Frage nach dem Demos}

Der Diskussionsbeitrag geht von einem die Neuzeit wesentlich bestimmenden Trend aus: Die wachsende Selbstverständlichkeit von Demokratie als pluralistischer Prozess - und von einer zunehmenden Inklusion, die in der Ausweitung der politischen Mitbestimmungsrechte auf davor ausgeschlossene Gruppen besteht.: Die letzten zwei Jahrhunderte waren durch die permanente Ausweitung des "Demos" in Richtung auf eine Deckungsgleichheit zwischen den an der Legitimierung politischer Macht Beteiligten und den dieser Macht Unterworfenen geprägt. Dieser Trend stößt aber deshalb auf Grenzen, weil die Erfahrungen neuzeitlicher Demokratie auf der territorialen Begrenztheit von als souverän fingierter Staaten aufbauen. Da aber die (vor allem ökonomische, aber auch kulturelle) Globalisierung die staatliche Souveränität und damit auch Territorialität zunehmend obsolet macht, verliert die demokratisch legitimierte Autorität der Staaten - und damit die Demokratie - immer mehr die Fähigkeit, Politik zu gestalten und insbesondere der globalisierten Ökonomie kontrollierend entgegen treten zu können.

Es geht dabei nicht um den Entwurf eines globalen Demokratiemodells, es geht - zunächst - um die Sensibilisierung für ein Problem, das in der anachronistischen Beschränktheit der Demokratie besteht. Eine solche Sensibilisierung reibt sich aber an der Ideologisierung des Volkes, des „Demos“. Dem Interesse einer demokratisch legitimierten Kontrolle von (vor allem auch) ökonomischer Macht steht die Idee von der Selbstverständlichkeit des „Demos“ entgegen. Diese tradierte Selbstverständlichkeit im Diskurs aufzubrechen, das ist die Aufgabe der Demokratietheorie im 2I. Jahrundert.

Die neuzeitliche Entwicklung der Demokratie ist durch zwei Trends gekennzeichnet, die den demokratietheoretischen Diskurs herausfordern und bestimmen: (Alonso et al. 20II; Müller 20II; Salzborn 20I2; Grande 2OI5)

- Aus den ethisch-normativen Entwürfen der Demokratie ist, mit vielen Rückschlägen, eine demokratische Realität entstanden, die zunehmend eine universelle Geltung beanspruchen kann. Die Entwicklung der politischen Ordnung der Neuzeit muss als Siegeszug der Demokratie gesehen werden. Das „We, The People of the United States" - zunächst eine Kampfansage einer durch ökonomische Privilegien ausgezeichneten bürgerlichen Elite an eine feudal-aristokratische Ordnung - hat sich in einem Systemtypus konkretisiert, der weltweit nahezu hegemonial ist.

- Der elitäre Charakter des Anspruchs auf Demokratie ausgedrückt in der Realität, dass 1776 eine kleine Gruppe weißer Männer, darunter „Besitzer" von Sklaven, in Anspruch nehmen konnten, „das Volk“ zu sein, ist von einem grundsätzlich universell akzeptierten Verständ- nis breitest möglicher Inklusion ersetzt worden. Prinzipiell ist im Gefolge internationaler Konventionen ein Verständnis von universellen Menschenrechten akzeptiert, die eine auf Differenzen des Geschlechts, der Religion, der politischen Überzeugung und der sexuellen Orientierung gegründete Exklusion vom "Demos“ als nicht akzeptable Diskriminierung wertet.

Ob diese Trends eher als Auflösung von Widersprüchen gesehen werden - wozu Francis Fukuyama neigt (Fukuyama 1992); oder ob sie ursächlich mit dem Entstehen neuer Widersprüche einher gehen - was die Sichtweise Samuel Huntingtons ist (Huntington 1996): Generell ist zu akzeptieren, dass Demokratie im zweiten Jahrzehnt des 2I. Jahrhunderts weltweit in einem signifikanten Maß breiter akzeptiert und umfassender verwirklicht ist als je zuvor, insbesondere auch in der ersten Hälfte des 20. Jahrhunderts, als Joseph Schumpeter Demokratie in einem die Demokratietheorie prägenden Sinn prozessual definiert (Schumpeter 1950) und, in der zweiten Hälfte des Jahrhunderts, als Robert Dahl einen rechtlich breit garantierten egalitären Zugang vieler zu den demokratischen Entscheidungen entwickelt hat. (Dahl 1989) Dahl hat so das auf den politischen Prozess abgestellte, in diesem Sinne formale Demokratieverständnis Schumpeters durch ein inhaltliches, normatives, egalitäres Konzept erweitert, das eine Beteiligung aller einer politischen Macht Unterworfenen zwingend vorsieht.

Der von Schumpeter und Dahl repräsentierte Doppelschritt dominiert das Demokratieverständnis der Gegenwart: Schumpeters am ökonomischen Marktmechanismus orientierte Definition von Demokratie als Wettbewerb um Wählerstimmen ist durch das Scheitern der marxistisch-leninistischen Antithese, Demokratie durch eine elitäre, aber einer generellen Gleichheit (Gerechtigkeit) verpflichteten Avantgarde herstellen zu lassen, im 2I. Jahrhundert nicht mehr umstritten. Demokratie setzt Pluralismus und politischen Wettbewerb voraus. Und Dahls kritische Ergänzung, dass der auf dem Prinzip individueller Freiheit aufbauende politische Markt der Demokratie durch einen garantierten und egalitären Zugang zu eben diesem Markt ergänzt werden muss (Dahls Konzept von „Citizenship“), ist ebenfalls nicht umstritten - mit einer freilich in ihrer Deutlichkeit immer klarer erkennbaren Ausnahme: Die Beschränkung des Zuganges zu "Citizenship“ als Voraussetzung des Zuganges zur Demokratie. (Bauböck 1994) 


\section{Inklusion - trotz Widerstandes}

Die Inklusion von Ausgeschlossenen folgte einem bestimmten Muster: Die zunächst durch ihre Inklusion Privilegierten setzten sich gegen die Ausweitung der Inklusion zu Wehr: gegen das Wahlrecht der armen weißen Männer am Beginn der Demokratieentwicklung in den USA; gegen das Wahlrecht der "freien“ (oder erst gerade von der Sklaverei befreiten) Afro-AmerikanerInnen in den USA; gegen das Wahlrecht und gegen die politische Gleichberechtigung von Frauen in der Verfassung der USA; gegen die bundesstaatliche Garantie des verfassungsrechtlich zugestandenen, aber in den Südstaaten der USA faktisch blockierten Wahlrechts der Afro-AmerikanerInnen - in Form der Bürgerrechtsgesetzgebung 1964, 1965. Die Strategie der Ausgeschlossenen war immer eine doppelte: auf der ethisch-normativen Ebene auf die grundsätzlich akzeptierten, de facto aber ignorierten Werte der Demokratie zu verweisen - auf das „We, The People“ oder die Menschenrechtsdeklaration; und faktisch, durch politischen Druck, die Interessen derer zu treffen, die vom Status quo profitierten. Es war das Bündnis von Idee und Interesse, das - bisher - die Demokratie weiter entwickelte: von ihren Anfängen als ein Luxusgut einer kleinen privilegierten Minderheit zu einem grundsätzlich alle Menschen inkludierenden System der Verteilung und der Kontrolle von Macht.

Lipset hat noch 1997 die Nation der USA („das Volk“) zweigeteilt gesehen. (Lipset 1997) Die Afro-AmerikanerInnen waren auch nach der Bürgerrechtsbewegung und deren gesetzlichen Erfolgen in den I960er Jahren eine gesellschaftlich weitgehend segregierte Sub-Nation. Das ist in der Zwischenzeit nicht generell anders geworden. Aber der Graben, der diese Subnation trennt, ist inzwischen weniger tief. Die Wahl Barack Obamas 2008 bedeutete kein Ende des Rassismus, kein Ende der alltäglichen Diskriminierung der „schwarzen“ Minderheit. Aber dass Obama von einer Regenbogenkoalition gewählt wurde, von einer Mehrheit aller politisch relevanter Gruppen der Gesellschaft, einschließlich der Mehrheit der Frauen - nur nicht von einer Mehrheit "weißer" Männer, das zeigt, dass der Prozess der Inklusion Fortschritte gemacht hat. (Hochschild/Weaver 20IO)

Die formelle Ausschließung von Personen wegen ihres Geschlechts, ihrer Herkunft, ihrer Überzeugungen und Präferenzen kann überwunden werden, ist weitgehend überwunden - mit Ausnahme eines Ausschlusses, der die Grenzen der Demokratieentwicklung vor Augen führt: Der Ausschluss aus dem Grund der fehlenden Staatsbürgerschaft. Dieser Ausschluss könnte, technisch gesehen, leicht überwunden werden: durch eine Garantie des Zuganges zur Staatsbürgerschaft nach transparenten Kriterien, die diesen Zugang nicht für eine längere Zeitspanne hinausschieben. Doch angesichts der mit dieser Frage verbundenen Migrationsbewegungen ist eine solche politische Lösung nicht zu erwarten. Denn die Migrationsbewegungen sind Teil der Globalisierung - und diese rüttelt an der Essenz des Modells nationalstaatlicher Souveränität.

\section{Der Verlust staatlicher Politikfähigkeit}

Der Ausschluss von Menschen aus dem Kreis der politisch Berechtigten aus dem Grund mangelnder Staatsbürgerschaft zeigt das Grundproblem einer Demokratie, die auf einem territorial begrenzten „Demos“ aufbaut. Das war zu Zeiten der Anfänge neuzeitlicher Demokratieentwicklung in den Schweizer Kantonen oder auch in den britischen Kolonien Nordamerikas weniger eine grundsätzliche Herausforderung als dies im 2I. Jahrhundert der Fall ist: Die Frage der Legitimation und der Kontrolle von Macht im ausgehenden I8. Jahrhundert betraf in Boston oder im Kanton Schwyz die vor allem von regionaler, von lokaler Macht Betroffenen. Die Globalisierung hat die territoriale Begrenzung der Macht - vor allem in ihrer ökonomischen Dimension gesprengt: BürgerInnen von Boston oder von Schwyz sind im 2I. Jahrhundert massiv von Ereignissen und Entscheidungen betroffen, die - wenn sie sich überhaupt lokalisieren lassen - in Schanghai oder Sao Paulo oder Frankfurt am Main zu verorten sind. Und damit ist das, was in Boston oder Schwyz - aber auch das, was in Wien oder Berlin entschieden wird - für die Frage nach der Legitimation und Kontrolle von Macht von abnehmender Bedeutung.

Die Nationalstaaten erfahren immer mehr, dass ihnen die Fähigkeit zur Politikgestaltung abhanden kommt. Die Ökonomie ist immer weniger von Wien oder Stockholm, aber auch immer weniger von London oder Paris zu steuern - wie auch die Fragen von Sicherheit immer weniger national zu beantworten sind. Die Politik ist dabei ihre Fähigkeit zu verlieren, der Macht der Ökonomie eine balancierende Gegenmacht entgegen stellen zu können. Und auch Kriege und Wanderungsbewegungen entziehen sich einer politischen Steuerung durch politische Macht, wenn diese staatlich und damit staatlich begrenzt verstanden wird.

Auf dieses Phänomen des staatlichen Politikverlustes gibt es eine rationale Antwort - das traditionelle Verständnis von Staatlichkeit aufzugeben; und das nicht aus primär normativen, sondern aus empirisch nachvollziehbaren Gründen. Der Staat als ein durch ein klar abgegrenztes Territorium und ein ebenso klar abgegrenztes (Staats-) Volk definiertes souveränes Gebilde, als Alpha und Omega der Politik, ist dabei, diese zentrale Rolle einzubüßen. Soll die Politik ihre Handlungsfähigkeit nicht mehr und mehr verlieren, müssen der Staat und seine Definitionsmerkmale überdacht werden: die territoriale Begrenztheit und die fiktive Eindeutigkeit 
des Volkes. Rainer Bauböck hat, im Anschluss an Robert Dahl, auf die Schere zwischen dem staatlichen Territorialitätsprinzip und der abnehmenden Politikfähigkeit verwiesen und auch die in Europa vorherrschende $\mathrm{Ab}$ lehnung der Doppelstaatsbürgerschaft kritisiert - als Widerspruch zu den Ansprüchen der Demokratie und dem Phänomen globaler Migration. (Bauböck 1994, 3-22, 197-205) Im Gefolge einer auch und wesentlich mit dem Phänomen "Globalisierung "verbundenen Relativierung der Bindungen an eine territorial definierte Staatlichkeit verliert der Staat zunehmend sein Monopol, politische Loyalitäten zu binden. Im 2I. Jahrhundert ist Staat nicht dasselbe wie im I9. Jahrhundert. Menschen sind immer weniger BürgerInnen, deren politisches Bewusstsein durch das Leben in einem einzigen Staat bestimmt ist.

Diese Option, die Politikfähigkeit zurückzugewinnen oder, besser, sie in einer transstaatlichen und transnationalen Form neu zu gewinnen, stößt auf einen derzeit und in absehbarer Zeit wohl nicht übersteigbaren Widerstand. Das sich nationalstaatlich definierende Volk ist nicht bereit, die Differenz zwischen Volk und Volk und damit zwischen Staat und Staat einfach am Altar einer rational konzipierten Transnationalität aufzugeben. Die Europäische Union als Versuchslabor von Transnationalität stößt in ihrer geplanten Vertiefung („An ever closer Union“ - Dinan 1999) auf einen massiven Euroskeptizismus, der sich auf alt-neue Nationalismen stützt und in den Erfolgen von anti-EU-Parteien äußert. (Leconte 20IO) Dieser Widerstand kommt oft von Parteigruppierungen, die (als "rechtspopulistisch") traditionell als politisch "rechts“ eingestuft werden. Er kommt aber auch von Gruppierungen, die sich als "links" verstehen und Transnationalität als Angriff auf den Sozialstaat verstehen - dessen Leistungsfähigkeit freilich nicht durch den Aufbau transnationaler politischer Strukturen sondern durch eine wachsende politische Hilflosigkeit des Nationalstaates bedroht ist. (Pelinka 2015)

Der sich im Wahlverhalten niederschlagende AntiGlobalisierungs-Affekt ist eine Erscheinung, die der von Theodor Adorno und anderen um die Mitte des 20. Jahrhunderts in den USA beobachtbaren Entwicklung einer „Autoritären Persönlichkeit" entspricht: In den USA waren es die ärmeren Schichten innerhalb der insgesamt privilegierten („weißen“) Mehrheitsbevölkerung, die um den Verlust ihres gesellschaftlichen Startvorteils fürchteten. Weil sie nichts zu verlieren hatten als das Privileg ihrer Hautfarbe, reagierten die ärmeren „weißen“ Männer („rednecks“) auf die sich abzeichnende Inklusion der „schwarzen“ Männer in einem signifikant überproportionalen Maße mit aggressiver Ablehnung. Aus einem Gefühl der Bedrohung heraus schotteten sich die ärmeren "Weißen“ gegen die afroamerikanische Minderheit ab. Als ideologischer Überbau diente ein Rassismus, der auf der behaupteten prinzipiellen Differenz zwischen "weiß" und „schwarz" baute. (Adorno et al. 1993)
Das analoge Phänomen im 2I. Jahrhundert ist die versuchte Abschottung gegen die Globalisierung. Die rechtspopulistischen Parteien Westeuropas unterscheiden sich von den rechtsextremen (faschistischen, nazistischen) Parteien des 20. Jahrhunderts durch eine weitgehend faktische Akzeptanz des demokratischen Prozesses und durch den auffallend ausgeprägten „proletarischen" Charakter ihrer aus den sozial schwachen Schichten kommenden Wählerschaft. (Wodak, KhosraviNik, Mral 2013) Deren politische Artikulation richtet sich gegen die, die sie als "fremd" wahrnehmen - und deren Integration in die Gesellschaft sie als Bedrohung empfinden: eine Bedrohung, die real ist - etwa auf dem Arbeitsmarkt; eine Bedrohung, die irreal ist - dann, wenn das „Anderssein“ der Fremden als objektive Gegebenheit konstruiert wird. In den USA ist der Aufstieg des Donald Trump die aktuelle Version dieser Entwicklung - zumeist ohne den um die Mitte des 20. Jahrhunderts noch offen artikulierten Rassismus, aber mit der gleichen Ausschließungstendenz gegenüber den als Bedrohung empfundenen und als "fremd“, als „anders" konstruierten Minderheiten. Diese Ausschließungsneigung ist funktional deckungsgleich mit dem Rassismus und seiner Rolle als Hindernis im Prozess der Demokratisierung. (Rosenberger, Mourao Permoser 2013)

Die Globalisierung macht staatliche Grenzen durchlässig und relativiert nationalstaatliche Souveränität. Macht verliert ihre nationale Zuordnung. Denn Macht findet statt, wenn Kapital grenzüberschreitend investiert wird; wenn Produktionsstätten von einem Staat in den anderen verlegt werden; und wenn Millionen Menschen wandern - und in Staaten wohnen, von deren politischer Macht sie abhängig sind, ohne dass sie die Politik dieser Staaten mit gestalten können. Demokratie als ein System der Legitimierung und Kontrolle von Macht bleibt hinter diesen Entwicklungen zurück. Die Herrschaft des durch staatliche Grenzziehungen eingegrenzten "Volkes“ entspricht immer weniger der Realität einer die Grenzen zwischen den Staaten aufhebenden Weltordnung.

Die Geschichte der universellen Demokratieentwicklung ist die Geschichte einer ständigen Erweiterung des "Demos" - und damit eine Geschichte der ständigen Neudefinition von Volk. Die Effekte der Globalisierung machen deutlich, dass die Geschichte der Demokratie als permanenter Erweiterung nicht an einem logischen Endpunkt angelangt ist. Die Definition des „Demos“ ist mehr denn je die Aufgabe der Demokratietheorie.

\section{Piketty? Ja, aber}

Demokratie baut auf Freiheit, und sie baut auf Gleichheit. Gleichheit hat aber mit Gerechtigkeit zu tun. (Rawls I97I) Das Freiheitspostulat der Demokratie be- 
trifft den Input Bereich des politischen Systems: Jeder und jedem muss die Freiheit garantiert werden, sich am politischen System zu beteiligen. Das Gleichheits- und Gerechtigkeitspostulat der Demokratie betrifft den Output Bereich des politischen Systems: Die Qualität einer Demokratie wird auch daran gemessen, wie groß das Ausmaß der durch Politik ermöglichten Reduzierung der (sozialen und ökonomischen) Ungleichheit ist.

Das größte Defizit an Gerechtigkeit ist - jedenfalls im 2I. Jahrhundert - die Schere zwischen den verschiedenen globalen Regionen. Die Indikatoren der menschlichen Entwicklung (Human Development Indicators) unterscheiden signifikant zwischen den ökonomisch entwickelten und den ökonomisch weniger entwickelten Regionen der Welt. Die Globalisierung beginnt jedoch diese Differenz zu verschieben: Sie führt zu wachsender Ungleichheit (oder: abnehmender Gerechtigkeit) in den entwickelten Regionen der Welt (Piketty 20I4) und inspiriert bzw. provoziert so die Affekte, die sich politisch gegen die Globalisierung richten. Pikettys kritische Analyse der wachsenden Differenz zwischen Arbeitseinkommen und Vermögen betrifft die Entwicklung in den reicheren Regionen der Welt insbesondere in Europa und Nordamerika. Aber gleichzeitig vermindert die Globalisierung das Entwicklungsgefälle zwischen den Regionen: Nach den Indikatoren ökonomischer und humaner Entwicklung sind die Nutznießer der Globalisierung die Gesellschaften in den ärmeren Regionen.

In seiner Studie über die Schwierigkeiten, die traditionell auf innerstaatliche Entwicklungen beschränkten Messungen von Ungleichheit (und Ungerechtigkeit) mit der abnehmenden Relevanz von staatlichen Grenzen in Einklang zu bringen, zeigt Steven Weisman das Ungenügen einer nur auf die innerstaatlichen Effekte gerichteten Debatte auf. (Weisman 20I6) Dass das rasante Wirtschaftswachstum in China die Ungleichheit in China selbst verstärkt hat - in Form der sich auseinander entwickelnden Einkommen, das ist die eine Seite der Realität; dass aber, erstens, die durch die generelle ökonomische Entwicklung Chinas die Lebensverhältnisse (gemessen etwa an der Gesundheitsversorgung, der durchschnittlichen Lebenserwartung, der Alphabetisierung) auch der armen Bevölkerungsschichten sich signifikant verbessert haben; und dass, zweitens, in einem globalen Vergleich "China's middle class" zu den großen Gewinnern der Globalisierung zählt, die „US lower middle class“ zu den großen Verlierern, das lässt eine Verallgemeinerung der Aussage einer primär Ungleichheit produzierenden Globalisierung ganz bestimmt nicht zu. (Weisman 2016, 49-65) Die Globalisierung hat eine wachsende Mittelschicht in den ärmeren Regionen der Welt zu den großen Gewinnern der Entwicklung gemacht.

Die pauschale Denunzierung der Globalisierung nimmt diesen Effekt nicht zur Kenntnis: Der rasante Rückgang des Analphabetismus in Asien und in Afrika; die ebenso rasante Steigerung der Lebenserwartung gerade auch in den ärmeren Regionen der Welt; die beginnende Problematisierung der prinzipiellen Ungleichbehandlung, der Diskriminierung von Frauen in den „Schwellenländern“ - das alles droht von einer Überempfindlichkeit der relativ Privilegierten in den privilegierten Regionen Europa und Nordamerika zugedeckt zu werden. Dass die Globalisierung auch und wesentlich eine Umverteilung von Wohlstand ist - zugunsten der Ärmeren - wird von der Fixierung auf die Befindlichkeit der sozial Schwächeren in den Reichtumsregionen verdrängt.

In der schrittweise erfolgten Inklusion der AfroAmerikanerInnen in den "Demos" der USA ist das $\mathrm{Zu}$ sammenspiel von struktureller Ungleichheit, Idee und Interesse zu beobachten. Gunnar Myrdals Studie „An American Dilemma" fasste eine Fülle von Daten zusammen, die rund um das Jahr 1940 erhoben waren. (Myrdal 1942) Die umfangreiche Studie belegte, dass die US-Gesellschaft insgesamt - trotz signifikanter Unterschiede zwischen dem Norden und Westen auf der einen, dem Süden der USA auf der anderen Seite - eine segregierte Gesellschaft war, in der die (im wesentlichen in den Südstaaten existierende) legale und die (überall zu beobachtende) faktische Diskriminierung der Afro-AmerikanerInnen mit einem Vorurteil einherging: Die Vorstellung von der "natürlichen“ Überlegenheit der „Weißen“ gegenüber den „Schwarzen“. In Myrdals Studie wird die "The Racial Beliefs of the Unsophisticated" genannt. (Myrdal I962, 97-IOI) und so der empirisch belegte $\mathrm{Zu}$ sammenhang zwischen Bildung („sophistication“) und Rassismus unterstrichen. Es waren vor allem die in der gesellschaftlichen Pyramide weiter unten platzierte, durch einen geringeren Bildungsstand und - damit zusammenhängend - geringeren sozialen Status charakterisierten „Weißen“, die um 1940, die von der Überlegenheit der eigenen „Rasse“ überzeugt waren.

Als 1962 Myrdals Studie neu aufgelegt wurde, diagnostizierte Arnold Rose in einem "Postscript" die in zwei Jahrzehnten eingetretenen Veränderungen: „There could be no doubt that the races were moving rapidly... (towards) desegregation by 1962." (Myrdal 1962, xliii) Das Parallelogramm der politisch und ökonomisch bestimmenden Interessen hatte sich verschoben - vor allem durch den Bedarf an qualifizierten Arbeitskräften. Die Idee einer Demokratie, die auf Freiheit und Gleichheit aller BürgerInnen beruhte, stand nun nicht mehr in dem 1940 beobachteten Ausmaß in Opposition zu den Interessen einer weißen Dominanzkultur, die vorhandene Startvorteile ideologisch absichern konnte. Durch die Bürgerrechtsgesetze der 1960er Jahre verschoben sich überdies Interessen, die den politischen Wettbewerb bestimmten: Das Gewicht „weißer" Stimmen, die nicht mehr von dem traditionellen Vorurteil eigener (natur- oder gottgegebenen) Überlegenheit ausgingen, in 
Verbindung mit dem wachsenden Stimmgewicht nicht"Weißer" Minderheiten reduzierte das politisch messbare Gewicht des US-amerikanischen Rassismus.

Eine analoge Konfliktsituation lässt sich im gegenwärtigen Konflikt zwischen einem „Demos“, der die Durchlässigkeit und Aufhebung staatlicher Grenzen als Bedrohung wahrnimmt, und den Folgen der Globalisierung beobachten. Aber im Gegensatz zu der Entwicklung in den USA fehlt eine verbindende normative Idee von der Zusammensetzung des „Demos“ - eine Idee, an der sich eine abweichende Realität reibt. Das herrschende Verständnis von dem, was Volk ist und sein soll, beruht nicht nur auf Inklusion, es beruht vor allem auch auf Exklusion. Das begründet die nationalstaatliche Beschränktheit der real existierenden Demokratie. Diese ist, solange sie nationalstaatlich verengt bleibt, strukturell gar nicht in der Lage, auf die globale Ungerechtigkeit und die globalen Verschiebungen zu reagieren. Die Demokratie in den reichen Regionen der Welt reagiert, als Folge der territorialen Begrenzung des „Demos“ und der demokratischen Prozesse in den als „souverän“ fingierten Staaten, auf die Umschichtung von Ungerechtigkeit aus der Sicht der Betroffenen in den reichen Staaten. Die Antworten auf einen Entwicklungsprozess, der durch die Eigendynamik der Globalisierung bewirkt wird, sind bestimmt von der Rücksichtnahme auf die Ängste Globalisierungs- und Modernisierungsverlierer in der reichen („ersten“) Welt. Diese Verlierer flüchten sich zunehmend in eine nationalistisch und rassistisch unterfütterte Defensivaggressivität, in eine zumindest latent militante Exklusion von „anderen“, von „Fremden“ - verbunden mit der Illusion, so die Globalisierung und ihre negativen Effekte verhindern zu können.

Diese im Rahmen der Nationalstaaten demokratisch legitimierte Abwehrhaltung entspricht der Abwehrhaltung der Maschinenstürmer, die im I9. Jahrhundert als Teil einer „radikalen Tradition“ Industrialisierung und Kapitalismus abwehren wollten (Hobsbawm 1999): Aus einem nachvollziehbaren Interesse stellten sie sich gegen Veränderungen, die ihren sozialen Status bedrohten. Anders als diese Maschinenstürmer der Vergangenheit sind die Maschinenstürmer der Gegenwart aber darüber informiert - oder könnten und sollten informiert sein welche Entwicklungen außerhalb ihrer sich potentiell zu Festungen entwickelnden nationalstaatlichen Demokratien vor sich gehen: etwa der Aufstieg von „Schwellenländern“ zu Großmächten oder das Massenmorden in globalen gesellschaftlichen Erdbebenzonen wie dem Nahen Osten.

Solange aber die Demokratie gefesselt ist an die scheinbar - Schutz bietende Territorialität des Nationalstaates findet sie darauf keine andere Antwort als die Umsetzung von Formeln wie „das Boot ist voll“. Solange die Demokratie auf einem Begriff von "Demos“ baut, der sich vor allem in der Abgrenzung zwischen Volk und
Volk definiert, besteht wenig Aussicht, dass die Demokratie andere Antworten als die $\mathrm{Ab}$ - und Ausgrenzung derer findet, die nicht zum (eigenen) „Demos“ gehören. Solange sich das eine Volk vor allem durch konstruiertes Anderssein und institutionelle Ein- und Ausschließung durch den Ausschluss des anderen Volkes definiert, verhindert die Demokratie in ihrer national verengten, beund eingegrenzten Form eine politische Globalisierung als Antwort auf die bereits bestehende ökonomische.

Die bestehende Demokratie in den (zum Beispiel europäischen) Nationalstaaten wird als Endpunkt der Demokratieentwicklung gesehen. Diese Wahrnehmung ignoriert, dass die Machverhältnisse, die zu legitimieren und zu kontrollieren die Kernaufgabe der Demokratie sind, sich nicht mehr auf die Etikette "französisch“ oder „polnisch“ reduzieren lassen. Ebenso ist die Differenzierung zwischen Innen- und Außenpolitik immer weniger realistisch: Die Wahl eines (einer) US-Präsidenten (Präsidentin) betrifft BürgerInnen Japans oder Brasiliens, ohne dass diese in irgendeiner Form die für sie relevante Entscheidung mit beeinflussen könnten. Die Weltpolitik wird zur Weltinnenpolitik - ohne dass die bestehenden politischen, die bestehenden demokratischen Strukturen dies reflektieren würden. (Büchele/Pelinka 20I3)

\section{Die Schwierigkeiten mit dem „Volk“}

Daher wäre es - unter Berücksichtigung der Inklusionsgeschichte der Demokratie - notwendig, die Begrifflichkeit „Volk“ zu überdenken. Das Resultat eines solchen Überdenkens kann nicht eine fertige Ordnung eines globalen politischen Systems sein, das alle WeltbürgerInnen zum globalen „Demos“ erklärt - das wäre noch naiver als die Vorstellung, dass Thomas Jefferson bei der Verfassung der US-amerikanischen Unabhängigkeitserklärung die Frauen, die "Native Americans“, die Afro-AmerikanerInnen in sein „We, The People“ hätte mit einschließen können. Aber die Inklusion der 1776 Ausgeschlossenen ist innerhalb von zwei Jahrhunderten grundsätzlich anerkannt und auch in konkrete Rechtsansprüche transferiert worden

Die Infragestellung des herrschenden Verständnisses (besser: der unterschiedlichen Formen des Verständnisses vom Volk) dient der Wahrnehmung des Widerspruchs zwischen Anspruch und Realität: zwischen dem Anspruch, dass in der Demokratie alle von Machtverhältnissen Betroffenen bei der Regelung dieser Verhältnisse mitwirken können; und der Realität einer Globalisierung, die alles zur Innenpolitik macht, ohne dafür Instrumente zur Mitbestimmung bereit zu stellen.

Jede Erweiterung der Inklusion reibt sich mit dem konkreten Verständnis von Volk. Die Geschichte der Definition von Volk ist die Geschichte der Spannung zwischen Inklusion und Exklusion, zwischen „us' and 
,them“'. Diese Geschichte ist durch eine Kontinuität von Aggression und Gewalt gekennzeichnet, die von „uns“ gegen „die anderen“ geübt wurde und mit dem Begriff von Volk und Nation gerechtfertigt wurde. Die Diktaturen des 20. Jahrhunderts haben sich auf das Volk berufen, auf eine mystische Volksgemeinschaft - und dabei eine den Demokratien analoge Begrifflichkeit verwendet.

Der zweite Satz des Bundes-Verfassungsgesetzes der Republik Österreich stellt fest, dass das Recht der Republik „vom Volk“ ausgeht. Was dieses Volk aber ist - die Summe aller im Staatsgebiet lebenden Menschen, oder die Summe der formell als BürgerInnen der Republik Anerkannten, war von Anfang an umstritten: Einer nüchtern-rationalen Definition stand eine ethnischrassistische entgegen. 192I entschied der deutschnationale Innenminister der Republik Österreich, Leopold Waber, dass „altösterreichische“ BürgerInnen aus dem nunmehr zu Polen gehörenden Galizien keinen Anspruch auf die Staatsbürgerschaft der Republik hätten, weil sie - obwohl deutschsprechend - als Jüdinnen und Juden keine Deutschen wären. (Judson 20I6, 445, 542) Diese Entscheidung war eine Vorwegnahme der Nürnberger „Rassen“-Gesetze und zeigte die Willkürlichkeit von Inklusion in das und Exklusion aus dem Volk.

Albert F. Reiterer hat die ethnische, die "völkische" Konnotation des Nationsbegriffes kritisch dekonstruiert. (Reiterer 1988) Um ein "Wir“-Gefühl im Einklang mit dem Verständnis universeller Menschenrechte zu begründen, braucht es die Akzeptanz des individuellen Ein- in und auch Austritts aus dem Volk. Und diese Akzeptanz ist von Wahrnehmungen und Interpretationen beeinflusst, die Inklusion und Exklusion mit dem Anschein von Konsistenz versehen: $\mathrm{Zu}$ "uns" gehört, von dem (der) wir annehmen, dass er (sie) „unsere“ Kultur akzeptiert, oder "unsere" Sprache spricht, oder die gleichen genetischen Wurzeln („roots“) aufweist, oder die gleiche Hautfarbe hat. Ein „Wir“-Gefühl ist immer auch von mehr oder weniger bewussten Rationalisierungen und Ideologisierungen von vorweg (als Vor-Urteil) bestehenden Ein- und Ausschließungen begleitet.

Hinter der Ideologisierung des Volkes und des damit verbundenen "Wir"-Gefühls war und ist immer der Gegensatz von Interessen erkennbar: Die „Haves“, die sich aus mehr oder weniger gut nachvollziehbaren Gründen von einem signifikanten Inklusionsschritt bedroht fühlen, stehen den „Have Nots“ gegenüber, die aus den analogen Gründen von einer Inklusion zu profitieren hoffen. Innerhalb eines grundsätzlich der Demokratie verpflichteten Systems führt das zu den Spannungen und Konflikten, von denen die Geschichte der US-amerikanischen Bürgerrechtsbewegung im I9. und vor allem im 20. Jahrhundert ebenso bestimmt war wie die Geschichte der Frauenbewegung. Innerhalb eines Systems, das - wie die Welt in ihrer Gesamtheit - aber keinerlei demokratische Strukturen aufweist, ist die Konfliktsitu- ation eine wesentlich andere: „Weltpolitik“ wird - etwa im Rahmen der Vereinten Nationen - von staatlichen Regierungen gemacht, die alles daran setzen, unter dem Stichwort "Nicht-Einmischung" die nationale Souveränität zu verteidigen; ein Prinzip, das zwar durch die ablaufenden vor allem ökonomischen Globalisierungsprozesse mehr und mehr ausgehöhlt wird, das offiziell aufzugeben aber schwer, ja unzumutbar erscheint: Lassen sich doch mit dem Anspruch, dieses Prinzip verteidigen zu müssen, Wahlen gewinnen - Wahlen in den abgeschotteten Bereichen der einzelnen Staaten.

Deshalb ist "global governance" nicht ausreichend, um den Widerspruch zwischen globalisierter Ökonomie und nationalstaatlich eingeengter Politik aufzulösen: Denn "global governance" als zwischenstaatliche Kooperationsmuster bleibt im Rahmen einer die staatliche Souveränitätsdoktrin respektierenden Ordnung. Erst die Herstellung supranationaler Strukturen - eben von "global government" - würde diesen Widerspruch vom Ansatz her aufheben. Doch solange das "Volk“ ausschließlich mit Bezug auf sich voneinander abgrenzenden Staaten verstanden wird, und solange die international relevanten Akteure die Interessen eines national und damit abgegrenzt definierten Volkes vertreten, ist "global government" nicht in Sicht.

"Global government" bedeutet den prinzipiellen Abschied von der Vorstellung nationaler Souveränität. Eben das sind die bisher beobachteten Versuche von "global governance“ - vor allem der Völkerbund und die Vereinten Nationen - eindeutig nicht. Was "global government" bedeuten müsste, lässt sich bei dem zentralen Diskurs über das Wesen der Europäischen Union erkennen: Es ist die Interpretationsschule des „NeoFunktionalismus", die in der Union ein Laboratorium von "transnational government" sieht - insbesondere im Europäischen Parlament, der Europäischen Kommission, dem Europäischen Gerichtshof, aber auch im Rat, wenn er nicht mehr an das Einstimmigkeitsprinzip gebunden ist. Die Debatte über den Demokratiecharakter der EU und über deren Bundesstaatlichkeit zeigt die Grenzen der politischen Durchsetzbarkeit von "transnational government" auf europäischer Ebene. (Schmitter 2000, Morgan 2005) Eben deshalb kann "global government" zwar als schlüssige Antwort auf den Politikverlust der Nationalstaaten konzipiert werden - etwa der institutionellen Ausgestaltung der EU folgend, aber auf absehbare Zeit sind die Chancen auf politische Umsetzung nicht gegeben.

\section{Die Kollision zweier Megatrends}

Demokratisierung war ein die letzten zwei Jahrhunderte dominierender Megatrend - methodisch und substantiell. Demokratie setzte sich immer mehr durch - metho- 
disch verstanden als Wettbewerb um Zustimmung von Betroffenen und Berechtigten; und inhaltlich verstanden als ständig fortschreitende Inklusion von Betroffenen, aber (noch) nicht Berechtigten. Im 2I. Jahrhundert ist die Demokratisierung mit einem anderen Megatrend konfrontiert - mit der Globalisierung. Die beiden Megatrends sind keineswegs inkompatibel. Aber die Weiterführung der Demokratisierung setzt voraus, dass das nationalstaatliche Axiom, das bisher die Demokratisierung bestimmt hat, zunächst überdacht, dann weiterentwickelt und schließlich relativiert wird. Wenn das nicht gelingt, wäre die Zukunft der Demokratie gefährdet: Denn dann würden Nationalstaaten, in eine prinzipielle Oppositionshaltung zur Globalisierung getrieben, zunehmend autoritäre Tendenzen entwickeln müssen beginnend mit der Infragestellung und partiellen Aufkündigung universeller Grundrechte.

Wie das Ergebnis der erkennbaren und wohl weiter zunehmenden Reibungskonflikte zwischen Demokratie und Globalisierung sein wird, muss natürlich offen bleiben. Aber es zeichnen sich zwei mögliche Szenarien ab: (Büchele/Pelinka 20I3, 45-76)

Das pessimistische Szenario: Der Widerstand der Interessen, die eine weitere Ent-Ethnisierung und eine Ent-Territorialisierung des "Demos" als Bedrohung ihrer relativ privilegierten Situation sehen, kann nicht auf dem Wege traditioneller nationalstaatlicher Demokratie aufgehoben werden. Der Widerstand führt zu einer Verschärfung einer "Das Boot ist voll“-Stimmung und einer Renaissance nationalstaatlicher Souveränitätsansprüche als Folge neo-nationalistischer Bewegungen und bei Wahlen erfolgreicher neo-nationalistischer Parteien. Ökonomisch führt dies zu mehr Protektionismus, politisch zu einem Rückzug in abgeschottete, eng umgrenzten Festungen. Da die Umverteilungswünsche der von diesen relativen Privilegierungen Ausgeschlossenen wachsen und auch an Aggressivität zunehmen werden, ist eine Zunahme von systematischer Gewalt die Folge: in Form traditioneller und nicht-traditioneller Kriege.

Das optimistische Szenario: Die in der gegebenen globalen Ordnung ökonomisch und kulturell relativ Privilegierten akzeptieren allmählich, dass die Sicherung ihrer gesellschaftlichen Position eine Öffnung nicht nur der ökonomischen sondern auch der politischen Systeme erfordert. Die von Inglehart schon Ende des 20. Jahrhunderts beobachteten Entwicklungstrends - der Zunahme von Bildung und Zunahme von Wohlstand - verstärken die Einsicht, dass auf die nicht mehr aufzuhaltende ökonomische Globalisierung eine politische Globalisierung folgen kann und soll: aus dem Eigeninteresse der relativ Privilegierten heraus. Es entstehen mehr und mehr transnationale Formen von Staatlichkeit. Die Europäische Union wird als Muster, als Instrument für die Rückgewinnung von Politikfähigkeit im Zeitalter der Globalisierung gesehen - als ein Schritt zu "global government“.
Die Idee einer die nationale Begrenztheit sprengenden Demokratie trifft sich mit dem Interesse einer von der Globalisierung profitierenden Gesellschaft.

Das optimistische Szenario setzt eine Akzeptanz von Strukturen eines "global government" voraus, die der Vorstellung nationalstaatlicher Souveränität widersprechen und diese in letzter Konsequenz aufheben. Das pessimistische Szenario schließt eine solche Entwicklung mit dem Hinweis auf eine Ablehnungsspirale aus: Die unvermeidlichen VerliererInnen einer Entwicklung hin zu "global government" sind - solange "global government" nicht existiert - mit einer Vetomacht ausgestattet. Die demokratisch legitimierten Regierungen der Nationalstaaten können ihren BürgerInnen, die ja ihre WählerInnen sind, nicht einfach Lösungen aufzwingen, die zwar als eine rationale und vor allem auch als eine substanziell demokratische Antwort auf die Globalisierung zu begründen sind, aber kurz- bis mittelfristig den BürgerInnen der privilegierten Zonen der Welt vor allem auch Nachteile bringen. Die als „Flüchtlingskri-

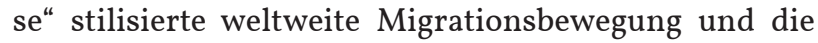
Reaktionen in Nordamerika und Europa, Japan und Australien zeigen, wie schwierig eine rationale Politik auf nationaler Ebene zu legitimieren ist. Das sich in der herrschenden Form der Demokratie vor allem, ja fast ausschließlich national artikulierende Interesse steht der Idee transnationaler Demokratie effektiv im Wege.

Die Vorstellung von einer globalen demokratischen Struktur als in sich logischen Umgang mit der ökonomischen Globalisierung und die von Defensivinteressen beherrschten demokratischen Strukturen der Staaten in den privilegierten Regionen der Welt sind nur schwer in Einklang zu bringen. Die Demokratietheorie, die mit der permanenten Aufhebung von Exklusion beschäftigt hat, ist gefordert, Konzepte zur Überwindung der im 2I. Jahrhundert dringlichsten Form der Verengung des "Demos“ zu beschäftigen: Mit der territorialen Dimension von Demokratie. Diese Beschäftigung kann sich auf ein Interesse stützen - auf das Interesse, Politikfähigkeit jenseits des Staates zu entwickeln. Und sie kann sich auf eine Idee stützen: Allen von konkreten Machtverhältnissen Betroffenen die Möglichkeit zur Mitsprache bei der Legitimierung, der Kontrolle, und der Veränderung solcher Machtverhältnisse einzuräumen.

\section{Literaturverzeichnis}

Adorno, Theodor et al. (1993). The Authoritarian Personality. New York.

Alonso, Sonia/John Keane/Wolfgang Merkel (eds.) (20II). The Future of Representative Democracy. Cambridge. Anderson, Benedict (2006). Imagined Communities. Reflections on the Origins and Spread of Nationalism, London. 
Bauböck, Rainer (1994). Transnational Citizenship and Rights in International Migration, Cheltenham.

Benhabib, Seyla (ed.) (1996). Democracy and Difference. Contesting the Boundaries of the Political, Princeton.

Büchele, Herwig/Anton Pelinka (2013). Weltinnenpolitik, Innsbruck.

Cannadine, Daviod (2013). The Undivided Past. Humanity beyond Differences. New York.

Dahl, Robert A. (1989). Democracy and Its Critics, New Haven.

Dinan, Desmond (1999). Ever Closer Union. An Introduction to European Integration. $2^{\text {nd }}$ edition, London

Fukuyama, Francis (1992). The End of History and the Last Man, New York.

Grande, Edgar (2015). Transformations of Democracy in a Globalized World, in: Volker Schneider/Burkhard Eberlein (eds.): Complex Democracy. Varieties, Crises, Transformations. Cham, 219-230.

Hobsbawm, Eric (1999). Uncommon People. Resistance, Rebellion and Jazz, London.

Hochschild, Jennifer/Vesla Mae Weaver (2010). There's No One as Irish as Barack O'Bama: The Policy of American Multiculturalism, in: Perspective on Politics. American Political Science Association, Vol 8(3), 737-759.

Huntington, Samuel P. (1996). The Clash of Civilizations and the Remaking of World Order, New York.

Inglehart, Ronald (1997). Modernization and Postmodenization. Cultural, Economic, and Political Changes in 43 Societies. Princeton.

Judson, Pieter M (2016). The Habsburg Empire. A New History. Cambridge, MA.

Leconte, Cécile (20IO). Understanding Euroscepticism, Houndsmill.

Lipset, Seymour Martin(1979). American Exceptionalism. A Double-Edged Sword, New York.

Morgan, Glyn (2005). The Idea of a European Superstate. Public Justification and European Integration, London.

Müller, Jan-Werner (20II). Contesting Democracy. Political Ides in Twentieth-Century Europe, New Haven.

Myrdal, Gunnar (1962). An American Dilemma. The Negro Problem and Modern Democracy. $2^{\text {nd }}$ edition. 2 volumes, New York.

Pelinka, Anton (2015). Die Unheilige Allianz. Die rechten und die linken Extremisten gegen Europa, Wien.

Piketty, Thomas (2014). Capital in the Twenty-First Century, Cambridge, MA.

Rawls, John (197I). A Theory of Justice, Cambridge, MA.

Reiterer, Albert F. (1988). Die unvermeidbare Nation. Ethnizität, Nation und nachnationale Gesellschaft, Frankfurt am Main.

Rosenberger, Sieglinde (1996). Geschlechter - Gleichheiten - Differenzen. Eine Denk- und Politikbeziehung, Wien
Rosenberger, Sieglinde/Julia Mourao Permoser (20I3). Zugehörigkeitspolitik - schmutziges Geschäft der politischen Grenzziehung? In: SWS-Rundschau 53(4), 43I-453.

Salzborn, Samuel (2012). Demokratie. Theorien, Formen, Entwicklungen, Baden-Baden.

Schmitter, Philippe C. (2000). How to Democratize the European Union... and Why Bother? Lanham (MD).

Schumpeter, Joseph A. (1950). Capitalism, Socialism and Democracy, New York.

Weisman, Steven R. (2016). The Great Tradeoff. Confronging Moral Conflicts in the Era of Globalization, Washington, DC.

Wodak, Ruth/Majid KhosraviNik/Brigitte Mral (eds), (2013). Right-Wing Populism in Europe. Politics and Discourse, London.

\section{Author}

Anton Pelinka is Professor of Nationalism Studies and Political Science at the Central European University, Budapest. From $1975-2006$ he was full professor at the University of Innsbruck, Austria and visiting professor at Harvard University (Schumpeter Fellow), Stanford University (Austrian Chair), University of Michigan, Ann Arbor, the University of New Orleans, and the Université Libre de Bruxelles (Institute for European Studies). His research fields include Comparative Politics and Democratic Theory. His immediate research focus will be on democracy and transnational politics. His research fields include Comparative Politics and Democratic Theory and transnational politics. Among his publications: Die unheilige Allianz. Rechte und linke Extremisten gegen Europa, Böhlau: Wien (2015) 
